\title{
PALINOLOGIA DE SEDIMENTOS DA BACIA DE SÃO PAULO, TERCIÁRIO DO ESTADO DE SÃO PAULO, BRASIL
}

\author{
Murilo Rodolfo de LIMA \\ Mário Sérgio de MELO \\ Armando Márcio COIMBRA
}

\begin{abstract}
RESUMO
Os depósitos terciários da Bacia de São Paulo, que abrangem sedimentos referidos às formações São Paulo e Itaquaquecetuba, mais recentemente têm sido reinterpretados como um conjunto sedimentar paleogeno que inclui sistema basal de leques aluviais e canais entrelaçados, sistema lacustre (em parte sincrônico com os anteriores) e sistema fluvial meandrante de topo. A interpretação da Formação Itaquaquecetuba (sistema fluvial entrelaçado) tem sido controvertida, sendo ora considerada como pertencente aos depósitos basais, ora como produto de reativação tectônica e remanejamento de sedimentos no Neogeno ou mesmo Quaternário.

O presente estudo compreendeu a análise palinológica de sedimentos provenientes não só dos depósitos de leques aluviais e do sistema lacustre (atribuídos à Formação São Paulo) como também do sistema de canais entrelaçados (atribuídos à Formação Itaquaquecetuba). As palinofloras encontradas, com vários taxa de valor estratigráfico e paleoclimático, são bastante homogêneas nas várias amostras, sugerindo idades e paleoclimas muito próximos.

A dominância de taxa oligocenos, ao lado de uns poucos tidos como eocenos ou mais antigos, sugere duas hipóteses: 1) possível remanejamento de sedimentos eocenos no Oligoceno; 2) os taxa considerados como eocenos teriam tido, na realidade, distribuição até o Oligoceno, o que implicaria a necessidade de correção do seu intervalo de distribuição.

A relativa abundância de taxa indicativos de clima mais frio (coníferas), ao lado da escassez daqueles mais típicos de clima quente e úmido, sugere vigência de clima relativamente frio durante a sedimentação. Entretanto, não foi possível precisar o efeito das diferenciações florísticas de altitude na assembléia polínica preservada.
\end{abstract}

\section{ABSTRACT}

The Tertiary sediments of the São Paulo Basin, which include the São Paulo and Itaquaquecetuba Formations, have been recently interpreted as a Paleocene sedimentary ensemble comprising: a basal alluvial fan to braided-stream system; a partially contemporaneous lacustrine system; a meandering fluvial system at the top of the sequence. The origin of the Itaquaquecetuba Formation (braided-channel system) is controversial, and it has been considered by different authors as having been associated with the basal deposits of the basin and as due to tectonic reactivation and sediment reworking during the Neogene or Quaternary.

This study comprises the palynological analysis of sediments from the alluvial fan, lacustrine and braided-stream systems (this latter attributed to the Itaquaquecetuba Formation); the respective palynofloras are homogeneous and include several taxa of stratigraphic and paleoclimatic value, suggesting very close ages and climates.

The dominance of Oligocene taxa together with only a few others considered as Eocene or older suggest two hypothesis: 1) possible reworking of Eocene sediments in the Oligocene; 2) the taxa previously considered as Eocene in age may have, in fact, lived until Oligocene, and so should have their distribution interval suitably corrected.

The relative abundance of taxa indicating colder climates (conifers) and the scarcity of others more typical of hot and wet climate suggests that sedimentation took place under cold conditions. However, it was not possible to establish precisely the influence of altitudinal floristic differentiations in the preserved palynological assemblage.

\section{INTRODUÇÃO}

A ocorrência de áreas descontínuas preenchidas por sedimentos continentais e costeiros cenozóicos é uma feição marcante na geologia da parte leste do Estado de São Paulo. Os fatores associados à gênese de tais acumulações são na verdade mais abrangentes, já que afetaram toda a região sudeste e parte da região sul do país. 
No Estado de São Paulo, tais acumulações de sedimentos aparecem em duas províncias geomorfológicas distintas de acordo com a divisão de ALMEIDA (1964): a Província Costeira e o Planalto Atlântico.

Na área da Província Costeira, o principal fator associado à sedimentação refere-se às variações glácio-eustáticas quaternárias. Os depósitos são representados pela Formação Cananéia (pleistocena) e cordões litorâneos mais jovens. Atingem maior expressão em área no litoral sul do Estado, limitando-se a planícies relativamente embutidas (como a de Caraguatatuba) no litoral norte.

Outras acumulações de sedimentos na área da Província Costeira apresentam como principal fator associado fases de tectônica rúptil terciária. É o caso da Formação Sete Barras (paleogena), Formação Pariqüera-Açu (neogena) e depósitos relacionados, no baixo vale do Rio Ribeira do Iguape.

$\mathrm{Na}$ área do Planalto Atlântico, o principal fator associado à sedimentação é sem dúvida a tectônica. É nesta província geomorfológica que se encontram as principais bacias que compõem o "Sistema de Rift da Serra do Mar" (ALMEIDA, 1976): as de Volta Redonda e Resende (RJ), Taubaté e São Paulo (SP) e Curitiba (PR). Apresentam forma de grabens e semigrabens com preenchimento continental (fluvial e lacustre) de idade paleogena a neogena. Os processos tectônicos formadores associam-se com reflexos tardios dos processos continentais que determinaram a abertura do Atlântico Sul (a partir do Mesozóico) e subseqüentes deslocamentos da placa Sul-Americana. Foram particularmente ativos durante o Paleogeno, sendo retomados em pulsos sucessivamente atenuados ao longo do Neogeno e Quaternário.

Ainda na área do Planalto Atlântico ocorrem acumulações supostamente neocenozóicas (pliopleistocenas), relativamente restritas em área, e associadas a encostas e vales atuais. Aparecem nos vales dos rios Jundiaí, Atibaia e Jaguari, com ocorrências importantes nas localidades de Tanque, Atibaia e Piracaia. Encontram-se mais distantes da linha de costa atual, relativamente às bacias tectônicas do Sistema de Rift da Serra do Mar. Os principais fatores genéticos associados à sua gênese são pouco conhecidos, podendo tratar-se de reflexos atenuados, em direção ao interior, dos eventos tectônicos manifestados principalmente ao longo da linha de costa.

O presente trabalho objetiva principalmente analisar, do ponto de vista palinológico, o preenchimento sedimentar da Bacia de São Pau- lo, incluída no Sistema de Rift da Serra do Mar e situada na área do Planalto Atlântico.

\section{A BACIA DE SÃO PAULO}

A Bacia de São Paulo situa-se no alto Rio Tietê, no Planalto Paulistano (subdivisão do Planalto Atlântico), que é nivelado pela Superfície do Alto Tietê $(700-1000 \mathrm{~m})$, de idade neogena (ALMEIDA, 1964). Apresenta forma irregular, sendo que os contatos dos sedimentos são mais regulares a NW e norte, onde são controlados por falhas pós-sedimentares de direção ENEWSW. Ao sul os contatos são erosivos, com contornos recortados.

A bacia está implantada numa região de articulação de blocos tectônicos, resultando numa geometria relativamente complicada. A espessura dos depósitos é variável, configurando-se depressões e altos tectônicos menores dentro do embaciamento maior.

O preenchimento sedimentar atinge $320 \mathrm{~m}$ de espessura, na área de Cumbica (IPT, 1986), sendo representado por depósitos fluviais de constituição variada (síltico-argilosos e arenosos) e termos fanglomeráticos grossos nas bordas. Depósitos argilosos de origem lacustre aparecem na região do bairro da Barra Funda, em posição centralizada dentro do embaciamento.

Os depósitos, tidos classicamente como terciários desde sua primeira descrição por PISSIS (1842), foram referidos como "argillas de São Paulo" por MORAES REGO (1930), “camadas de São Paulo"' por MORAES REGO (1933) e "Formação São Paulo" por MEZZALIRA (1962).

JUNQUEIRA (1969) descreveu aluviões mais antigos que os atuais na área do Rio Pinheiros, observados também ao longo do Rio Tietê, tendo sido denominados "aluviões antigos dos rios Pinheiros e Tietê" por SUGUIO \& TAKAHASHI (1970). Estes depósitos foram denominados "Formação Itaquaquecetuba" por COIMBRA et al. (1983), considerados mais novos que a Formação São Paulo.

MELO et al. (1986) consideraram que o preenchimento sedimentar da Bacia de São Paulo compreende quatro tipos de depósitos: depósitos fanglomeráticos (de leques aluviais); depósitos de transição entre leques e planície (incluindo lamitos de corridas de lamas distais); depósitos de planície fluvial (de sistemas entrelaçado, anastomosado e meandrante); depósitos lacustres. Estes quatro tipos foram interpretados como resultado da variação lateral de condições de leques aluviais-planície-lago, das porções mais marginais em direção às mais centrais da 
bacia. Na vertical, consideraram uma possível passagem de sistema fluvial entrelaçado a anastomosado na base para meandrante no topo da seqüência. A partir de idades indicadas por análises palinológicas (Eoceno Médio e Oligoceno Inferior), concluíram pela penecontemporaneidade para a sedimentação dos diferentes tipos de depósitos.

RICCOMINI (1989) reinterpretou a estratigrafia dos depósitos da Bacia de São Paulo à luz da hipótese de que as bacias do Sistema de Rift da Serra do Mar constituiriam inicialmente uma única depressão. Denominou os depósitos de leques aluviais e sistema fluvial entrelaçado a anastomosado de MELO et al. (1986) de Formação Resende, considerando-a como de leques aluviais e sistema fluvial entrelaçado, e os depósitos de sistema fluvial meandrante do topo da seqüência de Formação São Paulo; para os depósitos de sistema fluvial entrelaçado da área de Itaquaquecetuba manteve a designação de Formação Itaquaquecetuba. Considerou as formações Resende, Tremembé e São Paulo como pertencentes a uma seqüência paleogena (Grupo Taubaté), e a Formação Itaquaquecetuba como sendo neogena (ou ainda mais nova), com base na aparente relação dos depósitos desta unidade com fase tectônica do final do terciário (transcorrência sinistral neogena). Refutou as idades obtidas a partir de análises palinológicas anteriores indicando idade paleogena para a Formação Itaquaquecetuba (MELO et al., $1985 \mathrm{e}$ 1986; LIMA \& MELO, 1989; LIMA et al., 1989) por considerar o material analisado proveniente de magaclastos extraclásticos, hipótese esta levantada também por FITTIPALDI et al. (1989).

É neste contexto de relativa controvérsia sobre a cronologia das unidades e estratigrafia dos depósitos que preenchem a Bacia de São Paulo que foram realizados os estudos palinológicos aqui apresentados, os quais trazem subsídios para o esclarecimento das idades e relações entre as unidades, e também sobre o paleoclima vigente durante a sedimentação e evolução tectônica da região.

\section{PALINOLOGIA}

\subsection{Dados prévios}

Até o momento, a única publicação referente à Palinologia dos sedimentos da Bacia de São Paulo é a de MELO et al. (1985). Neste trabalho, os autores apresentam, de forma resumida, os resultados obtidos da análise de uma amostra representativa da Formação Itaquaquecetuba. Dezesseis tipos polínicos foram ilustrados, dos quais dois classificados, com dúvidas, a nível específico, dez a nível genérico* e os demais não identificados. A lista completa dos taxa ilustrados é a seguinte:

\section{ESPOROS}

\section{cf. Polypodiaceoisporites potoniei}

Esporo trilete indeterminado (tipo 1)

Esporo trilete indeterminado (tipo 2)

cf. Podocarpidites sp.

cf. Margocolporites vanwijhei

Periporites sp. 1

Periporites sp. 2

Periporites sp. 3

Tricolporites sp. 1

Tricolporites sp. 2

Tricolporites sp. 3

Tricolporites sp. 4

Echitriporites sp.

Syncolporites sp.

Pólen indeterminado $\mathrm{n} \% 1$

Pólen indeterminado n 2

Com base na presença de Margocolporites vanwijhei, os autores atribuíram à unidade em questão uma idade eocena, correlacionando-a com a Formação Resende, da bacia homônima, e com a Formação Tremembé, da Bacia de Taubaté.

\subsection{Proveniência das amostras estudadas}

Três amostras procedentes de sedimentos da Formação São Paulo e cinco da Formação Itaquaquecetuba foram aqui estudadas, estando posicionadas na figura 1 .

Das amostras da Formação São Paulo, uma é procedente da região de Itaquaquecetuba e duas são representativas da facies lacustre da unidade, tendo sido coletadas por ocasião de escavações do Metrô para construção da Estação Barra Funda.

As cinco amostras da Formação Itaquaquecetuba distribuem-se do seguinte modo: duas são procedentes da região de Itaquaquecetuba, tendo sido coletadas na seção-tipo da unidade, e três são procedentes de afloramento situado na extremidade oposta da bacia, na região de Barueri.

Todas as amostras foram processadas segundo técnica palinológica padrão adotada para clásticos finos. De cada uma delas foram montadas duas lâminas, que se encontram depositadas na coleção científica do DPE/IG-USP sob os números GP/4T-277 a GP/4T-292.

* Embora tenham sido utilizados epítetos genéricos, estes foram escritos com minúsculas e sem grifo, fazendo supor um uso informal, de caráter morfológico, e não sistemático. 
Fig. 1-ESBOCO DOS SEDIMENTOS TERCIÁRIOS

E FALHAS DE REATIVACÄO NA ÁREA

DA GRANDE SÃO PAULO

(mod. de Melo et al. 1986)

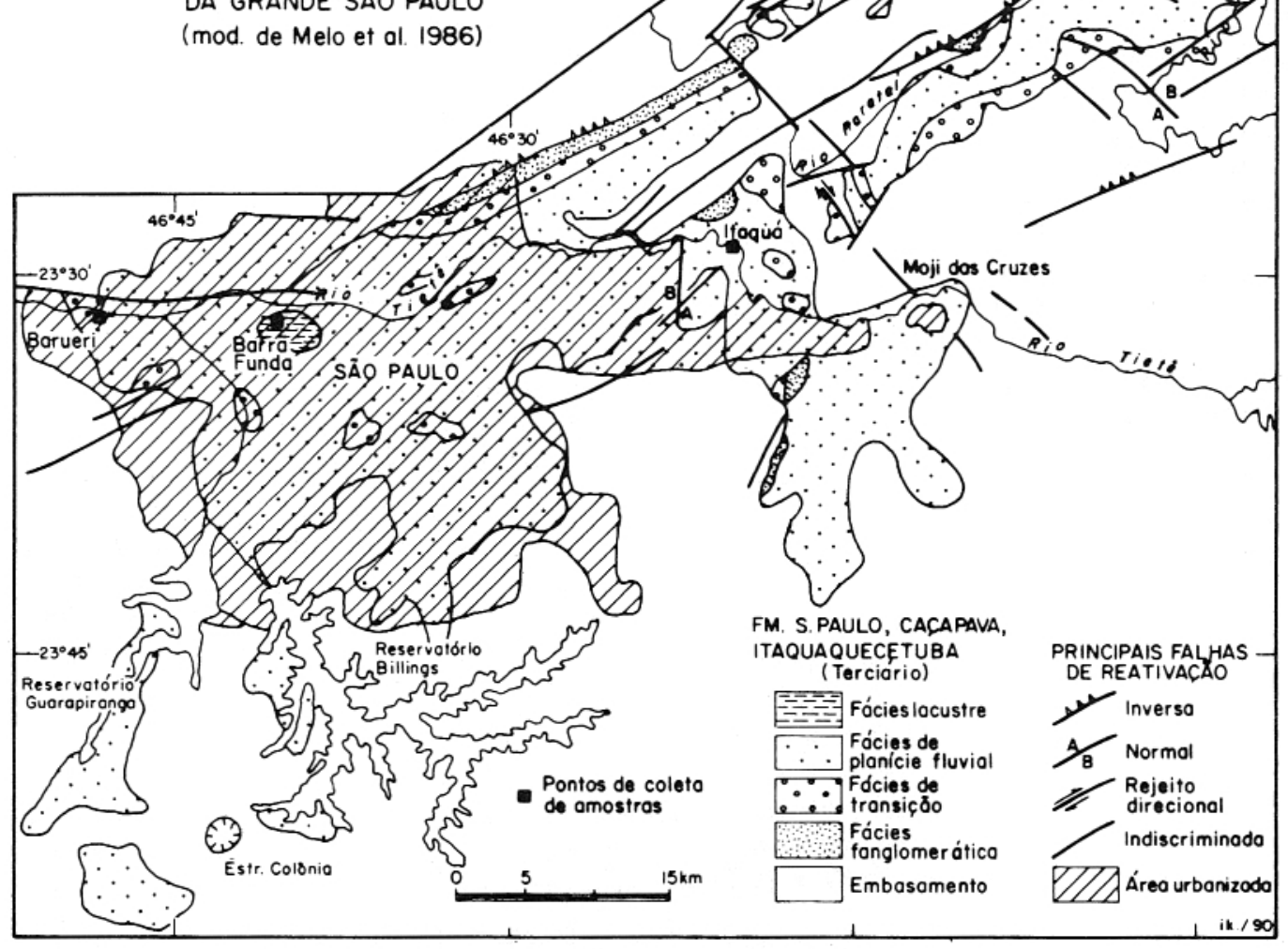

FIGURA 1 - Esboço dos Sedimentos Terciários e Falhas de Reativação na Área da Grande São Paulo (mod. de MELO et al., 1986). 
Rev. IG, São Paulo, 12(1/2), 7-20, jan./dez./1991

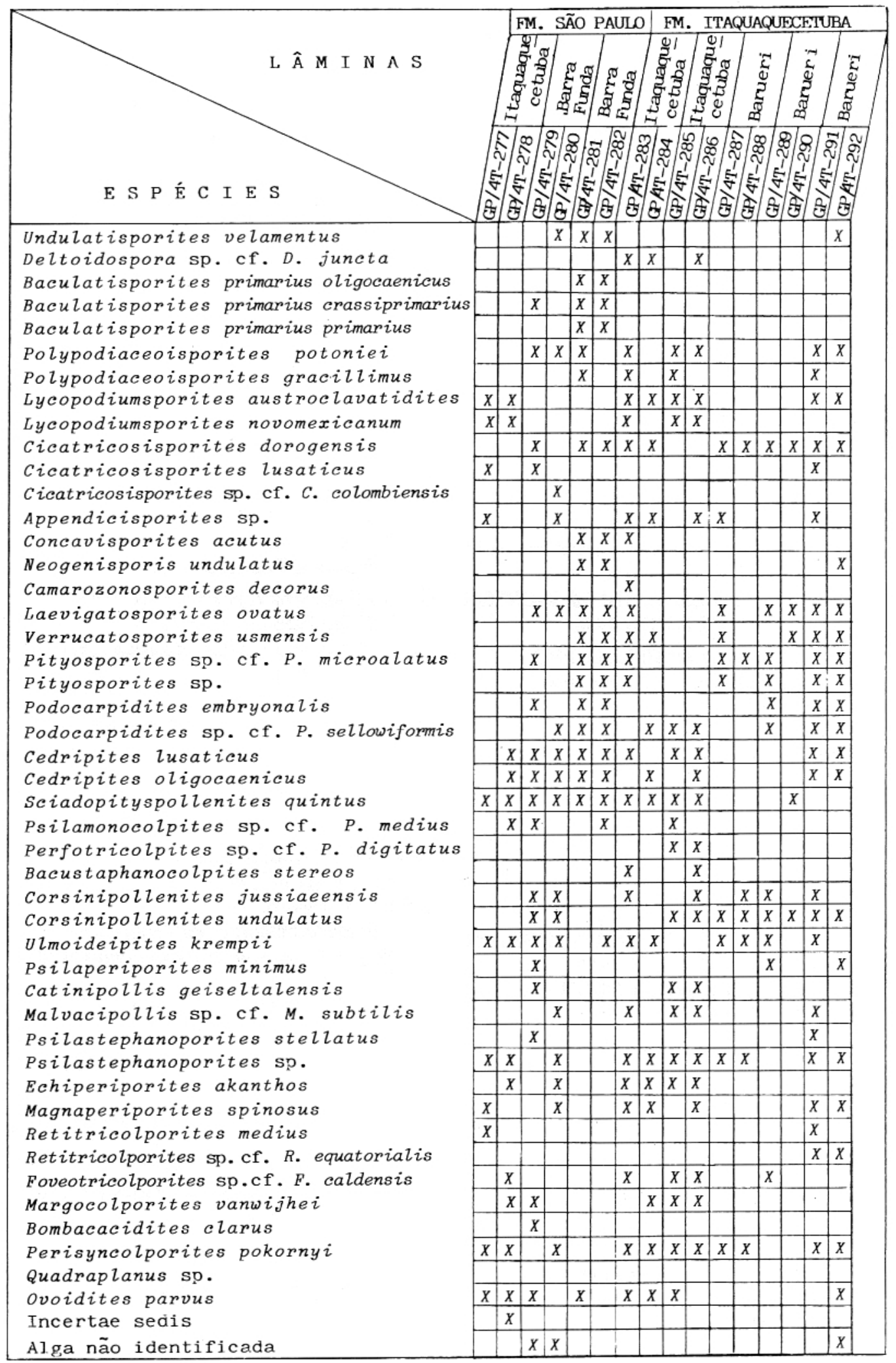

FIGURA 2 - Distribuição das espécies nas lâminas estudadas. 


\subsection{Taxonomia}

Integram a parte taxonômica do presente trabalho dezoito espécies de esporos, vinte e sete espécies de grãos de pólen, duas algas e um taxon considerado como "incertae sedis". Para suas identificações foram consultados, entre outros, os trabalhos de ANDERSON (1960), CHATEAUNEUF (1980), DUEÑAS (1980), GERMERAAD et al. (1968), GONZALEZGUZMÁN (1968), KRUTZCH (1963, 1967, 1971), LEIDELMEYER (1966), LIMA et al. (1985 a, b), REGALI et al. (1974 a, b), SAH (1967), SALARD-CHEBOLDAEFF (i981) e SCHULER \& DOUBINGER (1970). A lista completa das formas encontradas - cuja ocorrência, por lâmina, é mostrada na figura 2 - é a seguinte:

\section{SPORITES}

Anteturma Proximegerminantes POTONIÉ 1975

Turma Triletes (REINSCH 1981) POTONIÉ \& KREMP 1954

Subturma Azonotriletes LUBER 1935 emend. DETTMANN 1963

Infraturma Laevigati BENNIE \& KIDSTON 1886 emend. POTONIÉ 1956

Gênero Undulatisporites PFLUG 1953

Undulatisporites velamentus CHATEAUNEUF

Est. I, fig. 1

Afinidade botânica: Schizeaceae?

Gênero Deltoidospora MINER 1935 emend. POTONIÉ 1956

Deltoidospora sp. cf. D. juncta (KARA MURZA) ORLOWSKA-ZWOLINSKA

Est. I, fig. 2

Afinidade botânica: Lindsaya

Infraturma Apiculati BENNIE \& KIDSTON 1886 emend. POTONIÉ 1956

Subinfraturma Nodati DYBOWA \& JACHOWICZ 1957

Gênero Baculatisporites THOMSON \& PFLUG 1953

Baculatisporites primarius oligocaenicus KRUTZSCH

Est. I, fig. 3

Afinidade botânica: Osmunda

Baculatisporites primarius crassiprimarius KRUTZSCH

Est. I, fig. 4

Afinidade botânica: Osmunda

Baculatisporites primarius primarius KRUTZCH

Est. I, fig. 5

Afinidade botânica: Osmunda

Infraturma Murornati POTONIÉ \& KREMP 1954
Gênero Lycopodiumsporites (THIEGART 1938)

DELCOURT \& SPRUMONT 1955

Lycopodiumsporites austroclavatidites (COOKSON) POTONIÉ

Est. I, fig. 10

Afinidade botânica: Lycopodiaceae

Lycopodiumsporites sp. cf. L. novomexicanum ANDERSON

Est. I, fig. 11

Afinidade botânica: Lycopodiaceae

Gênero Cicatricosisporites POTONIÉ \& GELLETICH 1933

Cicatricosisporites dorogensis POTONIÉ \& GELLETICH

Est. I, figs. 12, 13

Afinidade botânica: Schizeaceae

Cicatricosisporites lusaticus KRUTZSCH

Est. I, fig. 14

Afinidade botânica: Schizeaceae

Cicatricosisporites $\mathrm{sp}$. cf. C. colombiensis KEDVES

Est. I, fig. 15

Afinidade botânica: Schizeaceae

Infraturma Tricrassati DETTMANN 1963

Gênero Camarozonosporites PANT 1954 ex POTONIÉ 1956

Camarozonosporites decorus (WOLFF) KRUTZSCH

Est. II, figs. 3, 4

Afinidade botânica: Lycopodiaceae

Subturma Zonotriletes WALTZ 1935 (in

LUBER \& WALTZ, 1938)

Infraturma Cingulati POTONIÉ \& KLAUS 1954 emend. DETTMANN 1963

Gênero Polypodiaceoisporites POTONIÉ 1956 Polypodiaceoisporites potoniei KEDVES

Est. I, figs. 6, 7

Afinidade botânica: Polypodiaceae

Polypodiaceoisporites gracillimus NAGY

Est. I, figs. 8, 9

Afinidade botânica: Polypodiaceae

Gênero Neogenisporis KRUTZSCH 1962

Neogenisporis undulatus KRUTZSCH

Est. II, fig. 2

Afinidade botânica: Gleicheniaceae

Infraturma Auriculati SCHOPF 1938

emend. DETTMANN 1963

Gênero Appendicisporites WEYLAND \& KRIEGER 1953

Appendicisporites sp.

Est. II, fig. 16

Afinidade botânica: Schizeaceae

Gênero Concavisporites PFLUG 1953

Concavisporites acutus THOMSON \& PFLUG

Est. II, fig. 1

Afinidade botânica: desconhecida 
Turma Monoletes IBRAHIM 1933

Subturma Azonomonoletes LUBER 1935

Infraturma Laevigatomonoletes DYBOWA \& JACHOWICZ 1957

Gênero Laevigatosporites IBRAHIM 1933

Laevigatosporites ovatus WILSON \& WEBSTER

Est. II, fig. 5

Afinidade botânica: Polypodiaceae

Infraturma Sculptatomonoletes DIBOWA \& JACHOWICZ 1957

Gênero Verrucatosporites PFLUG 1952

Verrucatosporites usmensis GERMERAAD et al.

Est. II, fig. 6

Afinidade botânica: Polypodiaceae

\section{POLLENITES}

Anteturma Variegerminantes POTONIÉ 1975

Turma Saccites ERDTMAN 1947

Subturma Disaccites COOKSON 1947

Gênero Podocarpidites COOKSON 1947 emend. POTONIÉ 1958

Podocarpidites embryonalis KRUTZSCH

Est. II, fig. 8

Afinidade botânica: Podocarpaceae

Podocarpidites sp. cf. P. sellowiformis ZAKLINSKAYA

Est. II, fig. 9

Afinidade botânica: Podocarpaceae

Gênero Pityosporites SEWARD 1914

Pityosporites sp. cf. P. microalatus KRUTZSCH

Est. II, fig. 10

Afinidade botânica: Pinus

Pityosporites sp.

Est. II, fig. 7

Afinidade botânica: Pinus

Gênero Cedripites WODEHOUSE 1933

Cedripites lusaticus KRUTZSCH

Est. II, fig. 11

Afinidade botânica: Cedrus

Cedripites oligocaenicus KRUTZSCH

Est. II, figs. 12, 13

Afinidade botânica: Cedrus

Gênero Sciadopityspollenites RAATZ 1937

Sciadopityspollenites quintus KRUTZSCH

Est. II, figs. 14, 15

Afinidade botânica: Sciadopitys

Turma Plicates NAUMOVA 1937-1939

Subturma Monocolpates IVERSEN \& TROELS-SMITH 1950

Gênero Psilamonocolpites MATHUR 1966

Psilamonocolpites sp. cf. P. medius (VAN

DER HAMMEN) VAN DER HAMMEN \&

\section{GARCIA DE MUTIS}

Est. II, fig. 16

Afinidade botânica: Palmae

Subturma Tricolpates IVERSEN \& TROELS-SMITH 1950

Gênero Perfotricolpites GONZALEZ-GUZMÁN 1967

Perfotricolpites sp. cf. $P$. digitatus GONZALEZ-GUZMÁN

Est. II, fig. 17

Afinidade botânica: Convolvulaceae

Subturma Polycolpates IVERSEN \& TROELS-SMITH 1950

Gênero Bacustephanocolpites GONZALEZGUZMÁN 1967

Bacustephanocolpites stereos GONZALEZGUZMÁN

Est. II, fig. 18

Afinidade botânica: Dicotyledoneae

Turma Poroses NAUMOVA 1937-1939

Subturma Triporates IVERSEN \& TROELS-SMITH 1950

Gênero Corsinipollenites undulatus (GONZALEZGUZMÁN) LIMA \& SALARDCHEBOLDAEFF

Est. III, fig. 2

Afinidade botânica: Onagraceae

Corsinipollenites jussiaeensis JAN DU CHÊNE et al.

Est. III, fig. 1

Afinidade botânica: Onagraceae

Subturma Stephanoporates IVERSEN \& TROELS-SMITH 1950

Gênero Ulmoideipites ANDERSON 1960 Ulmoideipites krempii ANDERSON

Est. III, fig. 3

Afinidade botânica: Ulmaceae

Gênero Psilaperiporites REGALI et al. 1974

Psilaperiporites minimus REGALI et al.

Est. III, fig. 4

Afinidade botânica: Chenopodiaceae

Gênero Catinipollis KRUTZSCH 1966

Catinipollis geiseltalensis KRUTZSCH

Est. III, fig. 5

Afinidade botânica: Craniolaria

Gênero Malvacipollis HARRIS 1965

Malvacipollis sp. cf. $M$. subtilis STOVER (in STOVER \& PARTRIDGE)

Est. III, fig. 6

Afinidade botânica: Malvaceae

Gênero Magnaperiporites GONZALEZGUZMÁN 1967

Magnaperiporites spinosus GONZALEZGUZMÁN

Est. III, fig. 19

Afinidade botânica: Malvaceae 
Gênero Echiperiporites VAN DER HAMMEN \& WIJMSTRA 1964

Echiperiporites akanthos VAN DER HAMMEN \& WIJMSTRA

Est. III, fig. 9

Afinidade botânica: Dicotyledoneae

Subturma Stephanoporates IVERSEN ET TROELS-SMITH 1950

Gênero Psilastephanoporites VAN DER HAMMEN emend. REGALI et al. 1974

Psilastephanoporites stellatus REGALI et al.

Est. III, fig. 8

Afinidade botânica: Malpighiaceae

Psilastephanoporites sp.

Est. III, fig. 7

Afinidade botânica: Malpighiaceae

Observação: Pode tratar-se de formas poradas de $P$. pokornyi GERMERAAD et al. Subturma Tricolporates IVERSEN \& TROELS-SMITH 1950

Gênero Foveotricolporites PIERCE 1961

Foveotricolporites sp. cf. F. caldensis GONZALEZ-GUZMÁN

Est. III, fig. 13

Afinidade botânica: Dicotyledoneae

Gênero Retitricolporites (VAN DER HAMMEN 1956) VAN DER HAMMEN \& WIJMSTRA 1964

Retitricolporites sp. cf. $R$. equatorialis GONZALEZ-GUZMÁN

Est. III, fig. 11

Afinidade botânica: Dicotyledoneae

Retitricolporites medius GONZALEZGUZMÁN

Est. III, fig. 12

Afinidade botânica: Dicotyledoneae

Gênero Margocolporites GERMERAAD et al. 1968

Margocolporites vanwijhei GERMERAAD et al.

Est. III, fig. 14

Afinidade botânica: Caesalpiniaceae

Gênero Bombacacidites COUPER 1958

Bombacacidites clarus SAH

Est. III, fig. 15

Afinidade botânica: Bombacaceae

Subturma Syncolporates IVERSEN \& TROELS-SMITH 1950

Gênero Perisyncolporites GERMERAAD et al. 1968

Perisyncolporites pokornyi GERMERAAD et al.

Est. III, fig. 10

Afinidade botânica: Malpighiaceae

Turma Jugates ERDTMAN 1947

Subturma Tetradites COOKSON 1947
Gênero Quadraplanus STOVER (in STO-

VER \& PARTRIDGE 1903)

Quadraplanus sp.

Est. III, fig. 16

Afinidade botânica: Leguminosae

ALGAE

Gênero Ovoidites POTONIÉ 1951

ex KRUTZSCH 1959

Ovoidites parvus (COOKSON \& DETT-

MANN) NAKOMAN

Est. III, fig. 18

Afinidade botânica: desconhecida

Alga não identificada

Est. III, fig. 20

Afinidade botânica: desconhecida

\section{INCERTAE SEDIS}

Est. III, fig. 17

Afinidade botânica: desconhecida

Observação: A forma aqui ilustrada pode corresponder a um grão de pólen em vista equatorial.

\section{DISCUSSÃO}

\subsection{Idade}

A sugestão de uma idade paleogena (Eoceno Superior) para a Formação Itaquaquecetuba foi inicialmente apresentada por MELO et al. (1985), modificando um conceito até então arraigado de que os sedimentos da Bacia de São Paulo seriam do topo do Terciário ou mesmo do Quaternário. Os resultados ora divulgados modificam ligeiramente essa atribuição, posicionando as duas unidades da Bacia (Formações São Paulo e Itaquaquecetuba) no Oligoceno, em função da ocorrência de Cicatricosisporites dorogensis, Verrucatosporites usmensis, Sciadopityspollenites quintus, Catinipollis geiseltalensis, Psilastephanoporites stellatus, Magnaperiporites spinosus e Margocolporites vanwijhei. A presença desta última espécie, que levou MELO $e t$ al. (op. cit.) a indicar idade eocena para os níveis portadores, pode ter dois significados diferentes: ou a espécie não é restrita ao Eoceno, alcançando o Oligoceno, ou representa um ciclo de retrabalhamento.

Do ponto de vista tectônico, os argumentos apontados por RICCOMINI (1989) para considerar a Formação Itaquaquecetuba como neogena parecem ainda inconclusivos, conforme já destacado por FITTIPALDI (1990), que discute as hipóteses de os megaclastos serem intraclásticos (penecontemporâneos da sedimentação) ou extraclásticos (pretéritos à sedimentação). 

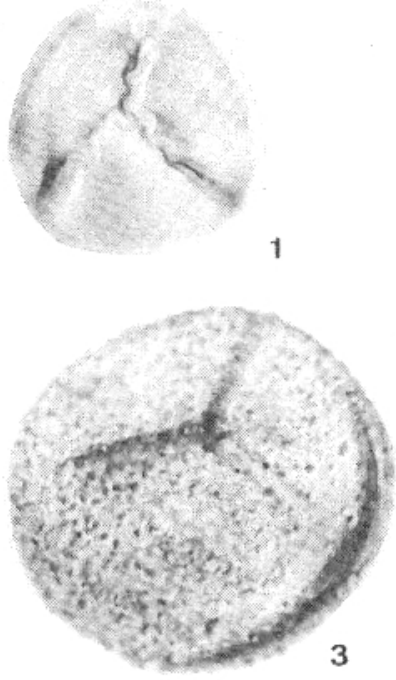

3

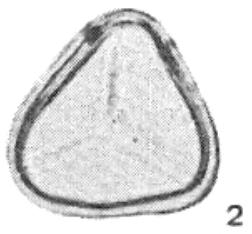

2

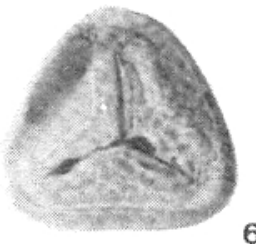

6

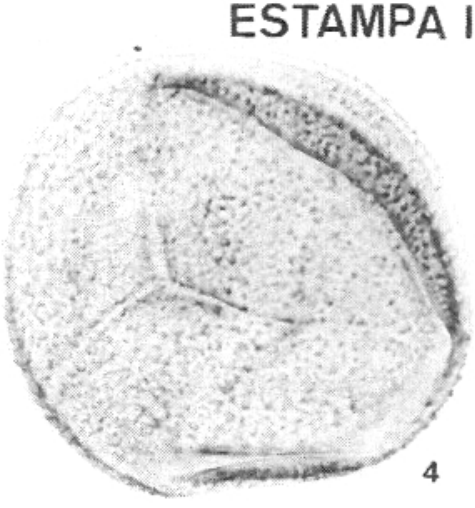

4
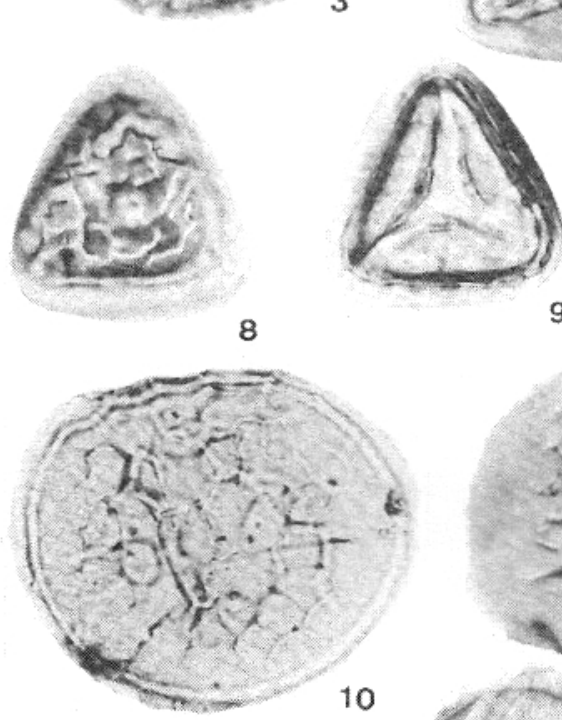

,

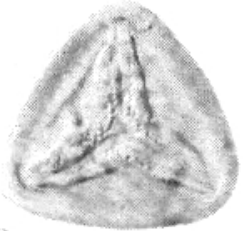

7
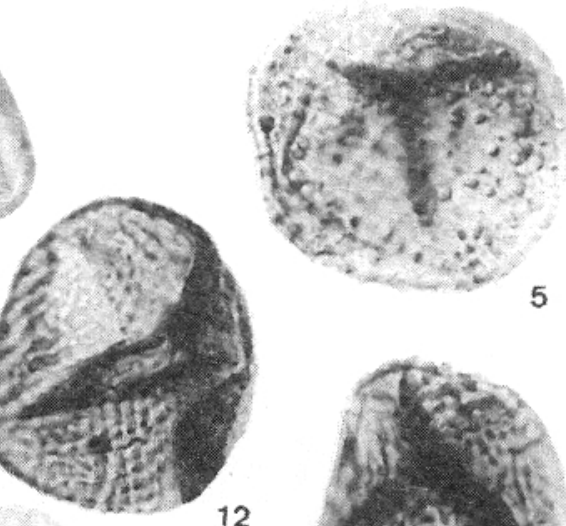

12

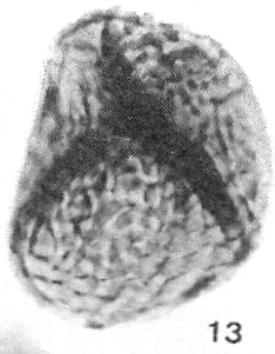

13
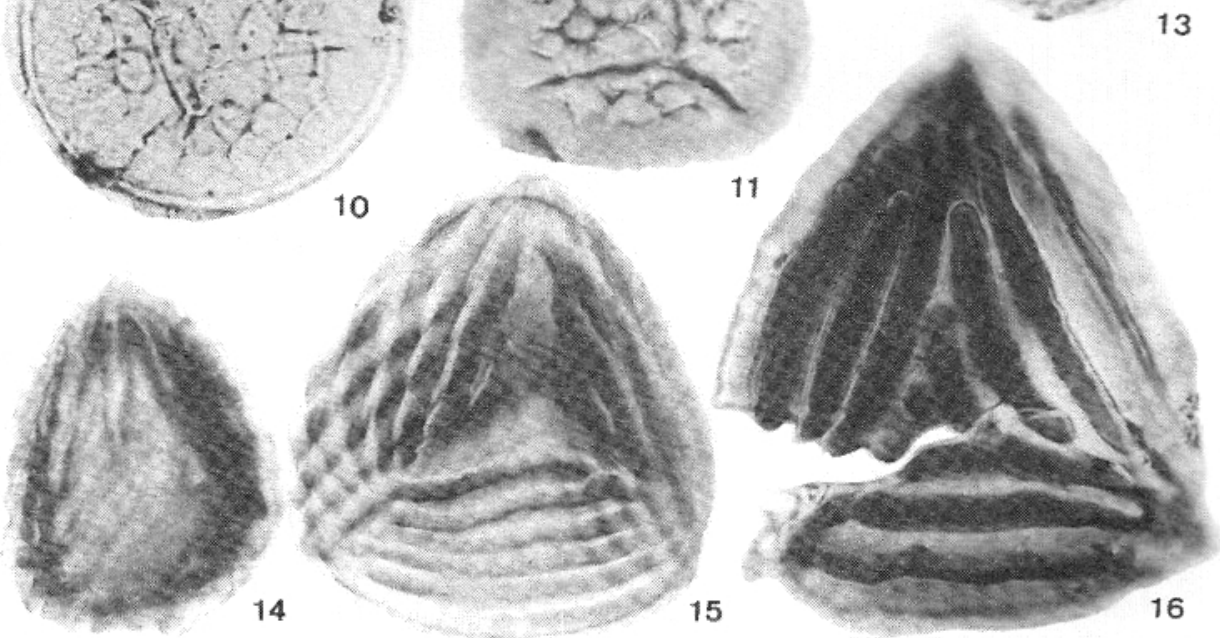

ESTAMPA I - Fig. 01 Undulatisporites velamentus. Lâm. GP/4T-282 - Fig. 02 Deltoidospora sp. cf. D. juncta. Lâm. GP/4T-286 - Fig. 03 Baculatisporites primarius oligocaenicus. Lâm. GP/4T-282 - Fig. 04 Baculatisporites primarius crassiprimarius. Lâm. GP/4T-282 - Fig. 05 Baculatisporites primarius primarius. Lâm. GP/4T-282 - Fig. 06 Polypodiaceoisporites potoniei. Lâm. GP/4T-281 - Fig. 07 Polypodiaceoisporites potoniei. Lâm. GP/4T-281 - Fig. 08 Polypodiaceoisporites gracillimus. Lâm. GP/4T-285 Fig. 09 Polypodiaceoisporites gracillimus. Lâm. GP/4T-285 - Fig. 10 Lycopodiumsporites austroclavatidites. Lâm. GP/4T-286 - Fig. 11 Lycopodiumsporites novomexicanum. Lâm. GP/4T-283 - Fig. 12 Cicatricosisporites dorogensis. Lâm. GP/4T-282 - Fig. 13 Cicatricosisporites dorogensis. Lâm. GP/4T-290 - Fig. 14 Cicatricosisporites lusaticus. Lâm. GP/4T-277 - Fig. 15 Cicatricosisporites sp. cf. C. colombiensis. Lâm. GP/4T-280 - Fig. 16 Appendicisporites sp. Lâm. GP/4T-286. 

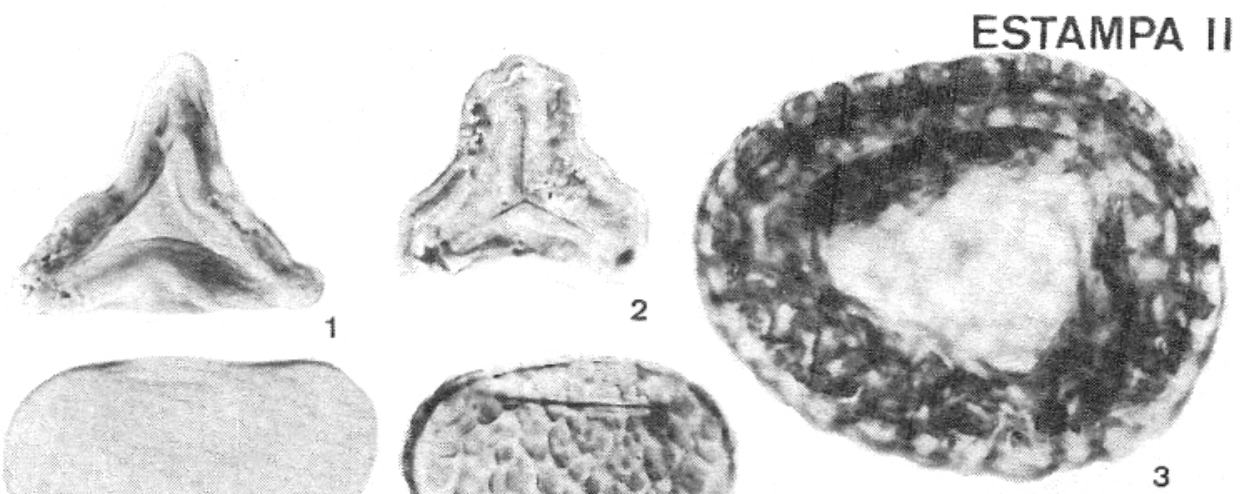

5

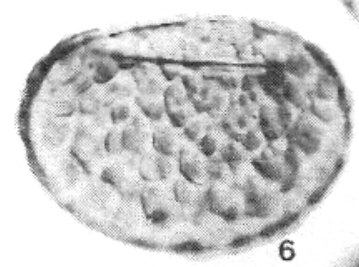

3
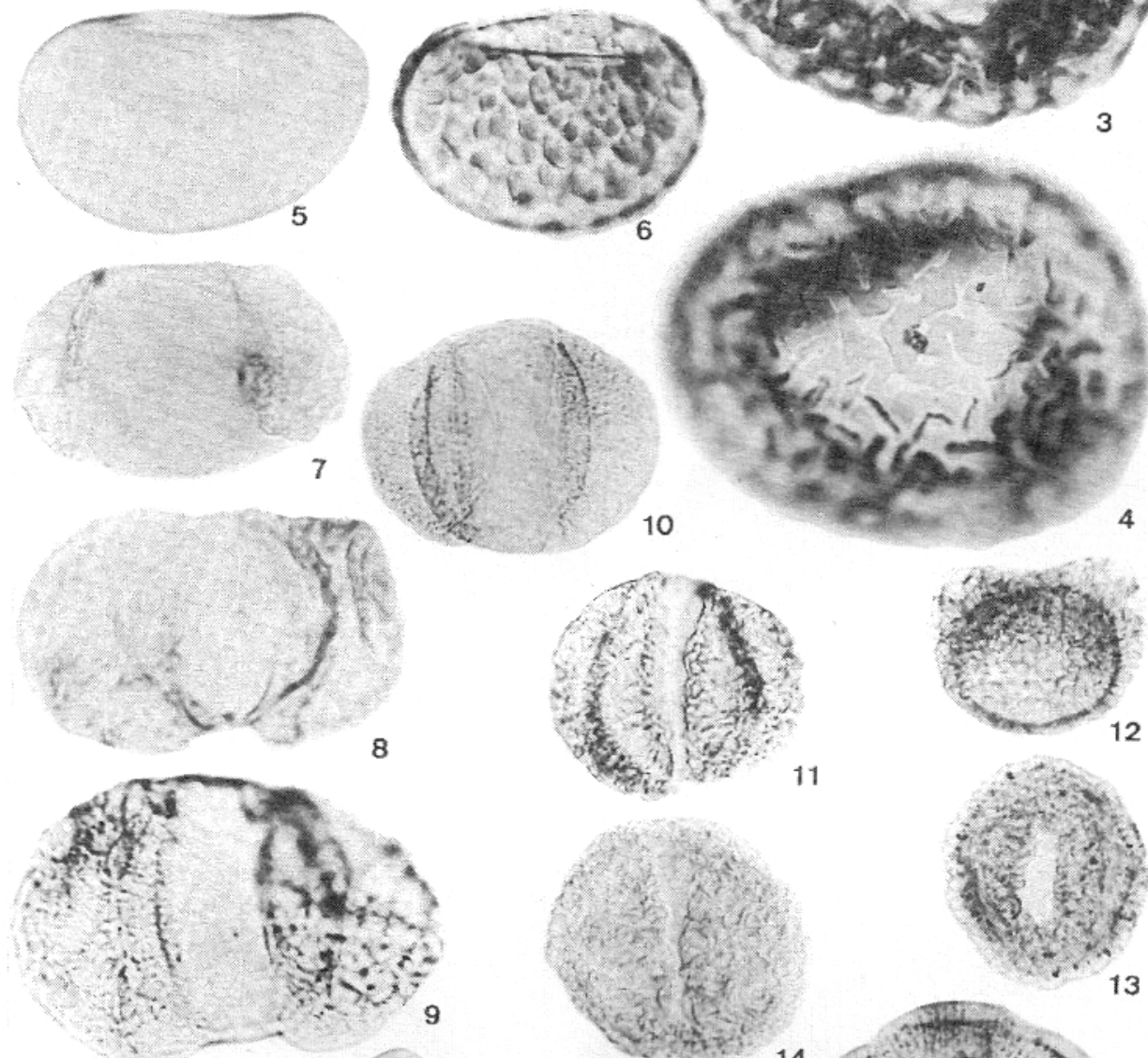

11
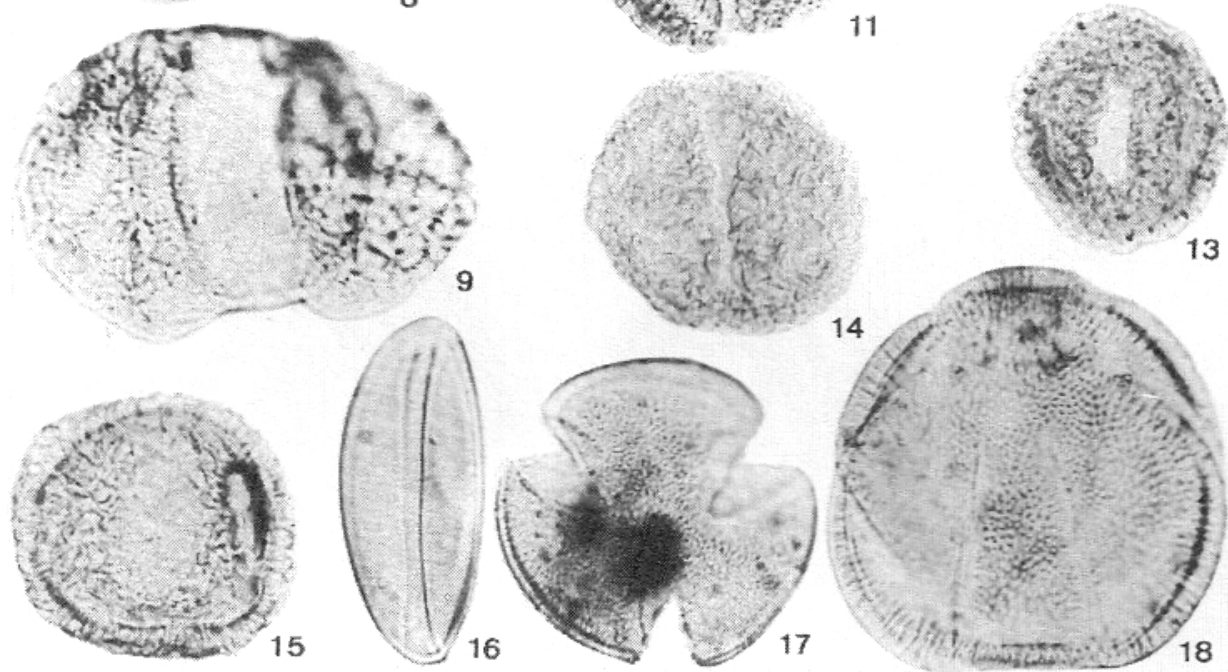

ESTAMPA II - Fig. 01 Concavisporites acutus. Lâm. GP/4T-283 - Fig. 02 Neogenisporis undulatus. Lâm. GP/4T-279 - Fig. 03 Camarozonosporites decorus. Lâm. GP/4T-279 - Fig. 04 Camarozonosporites decorus. Lâm. GP/4T-279 - Fig. 05 Laevigatosporites ovatus. Lâm. GP/4T-280 - Fig. 06 Verrucatosporites usmensis. Lâm. GP/4T-282 - Fig. 07 Pityosporites sp. Lâm. GP/4T-283 - Fig. 08 Podocarpidites embryonalis. Lâm. GP/4T-283 - Fig. 09 Podocarpidites sp. cf. P. sellowiformis. Lâm. GP/4T-285 - Fig. 10 Pityosporites sp. cf. P. microalatus. Lâm. GP/4T-282 - Fig. 11 Cedripites lusaticus. Lâm. GP/4T-281 - Fig. 12 Cedripites oligocaenicus. Lâm. GP/4T-281 - Fig. 13 Cedripites oligocaenicus. Lâm. GP/4T-282 - Fig. 14 Sciadopityspollenites quintus. Lâm. GP/4T-282 - Fig. 15 Sciadopityspollenites quintus. Lâm. GP/4T-282 - Fig. 16 Psilamonocolpites sp. cf. P. medius. Lâm. GP/4T-286 - Fig. 17 Perfotricolpites sp. cf. P. digitatus. Lâm. GP/4T-285 - Fig. 18 Bacustephanocolpites stereos. Lâm. GP/4T-286. 


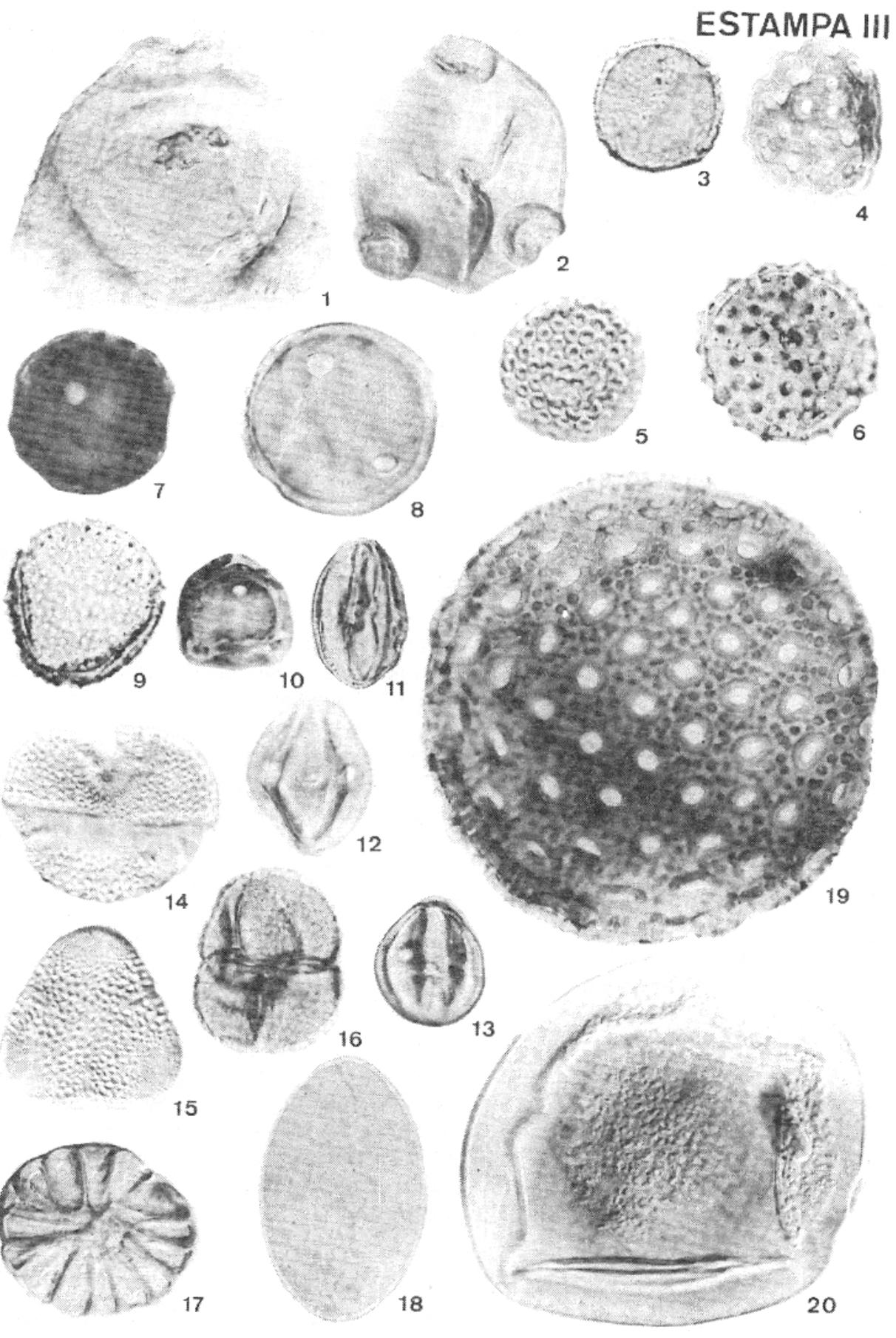

ESTAMPA III - Fig. 01 Corsinipollenites jussiaeensis. Lâm. GP/4T-283 - Fig. 02 Corsinipollenites undulatus. Lâm. GP/4T-279 - Fig. 03 Ulmoideipites krempii. Lâm. GP/4T-249 - Fig. 04 Psilaperiporites minimus. Lâm. GP/4T-279 - Fig. 05 Catinipollis geiseltalensis. Lâm. GP/4T-279 - Fig. 06 Malvacipollis sp. cf. M. subtilis. Lâm. GP/4T-280 - Fig. 07 Psilastephanoporites sp. Lâm. GP/4T-286 - Fig. 08 Psilastephanoporites stellatus. Lâm. GP/4T-279 - Fig. 09 Echiperiporites akanthos. Lâm. GP/4T-280 - Fig. 10 Perisyncolporites pokornyi. Lâm. GP/4T-291 - Fig. 11 Retitricolporites sp. cf. R. equatorialis. Lâm. GP/4T-277 - Fig. 12 Retitricolporites medius. Lâm. GP/4T-277 - Fig. 13 Foveotricolporites sp. cf. F. caldensis. Lâm. GP/4T-277 - Fig. 14 Margocolporites vanwijhei. Lâm. GP/4T-279 - Fig. 15 Bombacacidites clarus. Lâm. GP/4T-278 - Fig. 16 Quadraplanus sp. Lâm. GP/4T-285 - Fig. 17 Incertae sedis. Lâm. GP/4T-277 - Fig. 18 Ovoidites parvus. Lâm. GP/4T-279 - Fig. 19 Magnaperiporites spinosus. Lâm. GP/4T-280 - Fig. 20 Alga não identificada. Lâm. GP/4T-279. 
Por um lado, as cunhas clásticas associadas às falhas supostas neogenas incluem de fato megaclastos de depósitos tipo Itaquaquecetuba, o que vem confirmar que os depósitos eram inequivocamente preexistentes e já estavam litificados, à época da deformação tectônica. Por outro lado, tanto as análises palinológicas pretéritas (MELO et al., 1985 e 1986) como as do presente estudo foram realizadas também em material proveniente do local indicado como holoestratótipo da unidade, que, embora cortado por falhas pós-sedimentares, evidentemente não constitui um megaclasto. RICCOMINI (1989) admite que este material do holoestratótipo proviria de retrabalhamento de megaclastos extraclásticos pretéritos. Entretanto, o material palinológico ali contido não mostra evidências de retrabalhamento no Neogeno, tais como fragmentação de sedimentos preexistentes e associação de assembléias distintas.

Há que se considerar ainda que parte das feições interpretadas como megaclastos por RICCOMINI (1989) e FITTIPALDI (1990) possa corresponder, na realidade, a estruturas de corte e preenchimento, contendo material contemporâneo da sedimentação.

\subsection{Paleoecologia e paleoclima}

As amostras estudadas representam em geral subambientes particulares no contexto sedimentar das Formações São Paulo e Itaquaquecetuba. Há predominância de pequenos corpos de água pouco movimentados, que atuam como receptores da palinoflora local e, em menor escala, da vegetação presente na região, especialmente de espécies anemófilas. Estas observações são apoiadas pelo caráter síltico-argiloso das amostras, que atesta a relativa ausência de transporte dos sedimentos, bem como pela abundância đe matéria orgânica presente. A ocorrência de fungos (não identificados) e algas representa também uma evidência importante de condições estagnantes.

Várias das amostras são inteiramente dominadas, em termos quantitativos, pela flora local, constituída por esporos. Em alguns casos esses são de uma única espécie, como ocorre nas amostras procedentes de Barueri, nas quais se sobressai largamente a espécie Cicatricosisporites dorogensis (lâminas GP-287 a 292). O mesmo acontece também com as que provêm da Barra Funda, nas quais se destacam espécies do gênero Baculatisporites (lâminas GP/279 a 282). Dos elementos alóctones presentes, as angiospermas, cujas afinidades botânicas puderam ser atribuídas, correspondem em geral a famílias comumente representadas por formas arbustivas (convolvuláceas, quenopodiáceas, malpighiáceas, proteáceas, onagráceas, martiniáceas, malváceas, ulmáceas, entre outras) ou por árvores de comunidades abertas do tipo campo (Bombacáceas). Estas observações estão em aparente desacordo com as apresentadas por FITTIPALDI et al. (1989) e FITTIPALDI (1990) que, apesar de válidas apenas para a região de Itaquaquecetuba, ressaltam a presença de uma comunidade florestal, a partir da evidência fornecida por troncos e folhas. A observação de que o desacordo é aparente prende-se ao fato que, no caso da $\mathrm{Pa}$ linologia, várias famílias distintas produzem polens morfologicamente similares, como sucede nas formas tricolpadas e tricolporadas. Estas, cujas afinidades botânicas foram assinaladas como "Dicotyledoneae", podem portanto corresponder à comunidade arbórea portadora dos megafósseis mencionados.

A presença de grande variedade de coníferas - entre as quais representantes de Podocarpus, Pinus, Cedrus e Sciadopitys, em percentagens não negligenciáveis - sugere condições não tropicais a nível regional. Evidentemente, não pode ser descartada a possibilidade de que esta comunidade seja procedente de terras altas, possivelmente do Maciço do Itatiaia ou adjacências. A hipótese é contudo mais dificilmente aceitável que no caso da Bacia de Taubaté (LIMA et al., $1985 \mathrm{a}, \mathrm{b}$ ) pela maior distância relativa da Bacia de São Paulo da possível fonte dessa "contaminação".

\section{REFERÊNCIAS BIBLIOGRÁFICAS}

ALMEIDA, F.F.M. de. 1964. Fundamentos geológicos do relevo paulista. In: GEOLOGIA do Estado de São Paulo. São Paulo, Boletim 41 IGG. p. 169-263.

ALMEIDA, F.F.M. de. 1976. The system of continental rifts bordering the Santos Basin, Brazil. An. Acad. bras. Ciên., Rio de Janeiro, 48(Supl.):15-26.

ANDERSON, R. 1960. Cretaceous-Tertiary palynology, eastern side of the San Juan Basin, New Mexico. State Bur. Mines Miner.
Resour. Mem. 6:1-59.

CHATEAUNEUF, J.J. 1980. Palynostratigraphie et paléoclimatologie de l'Eocene Supérieur et de l'Oligocene du Bassin de Paris (France). Mém. B.R.G.M. 116:1-357.

COIMBRA, A.M.; RICCOMINI, C.; MELO, M.S. de. 1983. A Formação Itaquaquecetuba: evidências de tectonismo no Quaternário paulista. In: Simpósio Regional de Geologia, 4, São Paulo, 1983. Atas... São Paulo, SBG, p. 253-266. 
DUEÑAS, H. 1980. Palynology of OligoceneMiocene strata of borehole Q-E-22, Planeta Rica, northern Colombia. Rev. Paleobot. Palynol. 30(3/4):313-328.

FITTIPALDI, F.C. 1990. Vegetais fósseis da Formação Itaquaquecetuba (Cenozóico, Bacia de São Paulo). São Paulo (Tese de Doutoramento, Instituto de Geociências, USP).

FITTIPALDI, F.C.; SIMÕES, M.G.; GIULIETTI, A.M.; PIRANI, J.R. 1989. Fossil plants from the Itaquaquecetuba Formation (Cenozoic of the São Paulo Ba$\sin$ ) and their possible paleoclimatic significance. Boletim IG, Publicação Especial, 7, p. 183-203.

GERMERAAD, J.H.; HOPPING, C.A. \& MULLER, J. 1968. Palynology of Tertiary sediments from tropical areas. Rev. Paleobot. Palynol. 6:189-348.

GONZALEZ-GUSMÁN, A.E. 1967. A palynological study of the upper Los Cuervos and Mirador Formations (lower and middle Eocene, Tibu area, Colombia). Ed. Brill, Leyden, $68 \mathrm{p}$.

INSTITUTO DE PESQUISAS TECNOLÓGICAS DO ESTADO DE SÃO PAULO IPT - 1986. Tectônica e sedimentação cenozóica na área da soleira entre as bacias de São Paulo e Taubaté, São Paulo, IPT (Rel. 23724).

JUNQUEIRA, C.B. 1969. Camadas cruzadas de areias, gravas e cascalhos fluviais do Vale do Pinheiros, Campus da Cidade Universitária. São Paulo, Instituto de Geografia/USP, p. 7-9 (Notas Prévias 101).

KRUTZSCH, W. 1963. Atlas der mittel-und jungtertiären dispersen Sporenund Pollensowie der Mikroplanktonformen des nördlichen Mitteleuropas. Lieferung II. VEB Deutscher Verlag der Wissenschaften, $141 \mathrm{p}$.

KRUTZSCH, W. 1967. Atlas der mittel-und jungtertiären dispersen Sporenund Pollensowie der Mikroplanktonformen des nördlichen Mitteleuropas. Lieferung IV und V. VEB Deutscher Verlag der Wissenschaften, $232 \mathrm{p}$.

KRUTZSCH, W. 1971. Atlas der mittel-und jungtertiären dispersen Sporenund Pollensowie der Mikroplanktonformen des nördlichen Mitteleuropas. Lieferung VI. VEB Deutscher Verlag der Wissenschaften, $234 \mathrm{p}$.

LEIDELMEYER, P. 1966. The Paleocene and Lower Eocene pollen flora of Guyana. Leidse Geol. Meded. 38:49-70.

LIMA, M.R.; SALARD-CHEBOLDAEFF, M. \& SUGUIO, K. 1985a. Étude palynologique de la Formation Tremembé, Tertiaire du Bassin de Taubaté (État de São Paulo, Brésil) d'après les échantillons du sondage no 42 du CNP. MME-DNPM, sér. Geologia no. 27, Paleont./Estratig. no 2:379-393.

LIMA, M.R.; VESPUCCI, J.B.O. \& SUGUIO,

K. 1985b. Estudo palinológico de uma camada de linhito da Formação Caçapava, Bacia de Taubaté, Terciário do Estado de São Paulo, Brasil. An. Acad. brasil. Ciênc. 57(2):183-197.

LIMA, M.R. de \& MELO, M.S. de. 1989. Palinologia de sedimentos da Bacia de São Paulo. In: Workshop Geologia da Bacia de São Paulo, São Paulo. 1989. Coletânea das Comunicações... São Paulo, SBG/NSP, p. 35-37.

LIMA, M.R. de; MELO, M.S. de; COIMBRA, A.M. 1989. Palinologia de sedimentos da Bacia de São Paulo, Terciário do Estado de São Paulo, Brasil. II - A Formação Itaquaquecetuba. In: Congresso Brasileiro de Paleontologia, 11, Curitiba, 1989. Resumo das Comunicações... Curitiba, SBP, p. 96.

MELO, M.S. de; VINCENS, A.; TUCHOLKA, P. 1985. Contribuição à cronologia da Formação Itaquaquecetuba - SP. An. Acad. brasil. Ciênc., Rio de Janeiro, 57(2):175-181.

MELO, M.S. de; CAETANO, S.L.V.; COIMBRA, A.M. 1986. Tectônica e sedimentação na área das bacias de São Paulo e Taubaté: In: Congresso Brasileiro de Geologia, 34, Goiânia, 1986, Anais... Goiânia, SBG, 1:321-336.

MEZZALIRA, S. 1962. Novas ocorrências de vegetais fósseis cenozóicos no Estado de São Paulo. O IGG, 15:73-94.

MORAES REGO, L.F. de. 1930. A geologia do petróleo no Estado de São Paulo. Bol. Serv. Geol. Min. do Brasil, Rio de Janeiro, (46): $1-110$.

MORAES REGO, L.F. de. 1933. As formações cenozóicas de São Paulo. Anuário da Esc. Polit. S. Paulo, 2:231-267.

PISSIS, A. 1842. Mémoire sur la position géologique des terrains de la partie australe du Brésil et sur les soulèvements qui, à diverses époques, ont changé le relief de cette contrée. C.R. Acad. Sci. Paris, 14:353-413.

REGALI, M.S.P.; UESUGUI, N. \& SANTOS, A.S. 1974a. Palinologia dos sedimentos Meso-Cenozóicos do Brasil. Parte I. Bol. Tecn. Petrobrás 17(3):177-191.

REGALI, M.S.P.; UESUGUI, N. \& SANTOS, A.S. 1974b. Palinologia dos sedimentos Meso-Cenozóicos do Brasil. Parte II. Bol. Tecn. Petrobrás 17(4):263-301.

RICCOMINI, C. 1989. O Rift Continental do Sudeste do Brasil. São Paulo (Tese de Doutoramento, Instituto de Geociências, USP). 
SAH, S.C.D. 1967. Palynology of an Upper Neogene profile from Rusizi valley (Burundi). Mus. Midden-Afr. Ann., Reeksin $8^{\circ}$, Geol. Wetensch., 57:173p.

SALARD-CHEBOLDAEFF, M. 1981. Palynologie Maestrichtienne et Tertiaire du Cameroun. Resultats botaniques. Rev. Paleobot. Palynol. 32(4):401-439.
SCHULER, M. \& DOUBINGER, J. 1970. Observations palynologiques dans le Bassin d'Amaga (Colombie). Pollen et Spores 12(3): 429-450.

SUGUIO, K. \& TAKAHASHI, L.I. 1970. Estudo dos aluviões antigos dos rios Pinheiros e Tietê, SP. An. Acad. brasil. Ciên., Rio de Janeiro, 42(3):555-570.

Endereço dos autores:

- Murilo Rodolfo de Lima e Armando Márcio Coimbra - Universidade de São Paulo - Instituto de Geociências - Caixa Postal 20.899 - 01498 - São Paulo, SP - Brasil.

- Mário Sérgio de Melo - Instituto de Pesquisas Tecnológicas do Estado de São Paulo - IPT - Caixa Postal 7.141 05508 - São Paulo, SP - Brasil. 